\title{
Techniques for Cytologic Sampling of Pancreatic and Bile Duct Lesions
}

\author{
William Brugge, M.D., ${ }^{1 \star}$ John DeWitt, M.D., ${ }^{2}$ Jason B. Klapman, M.D. ${ }^{3}$ \\ Raheela Ashfaq, M.D., ${ }^{4}$ Vinod Shidham, M.D., ${ }^{5}$ David Chhieng, M.D., ${ }^{6}$ \\ Richard Kwon, M.D., ${ }^{\prime}$ Zubair Baloch, M.D., ${ }^{8}$ Matthew Zarka, M.D., \\ and Gregg Staerkel, M.D. ${ }^{10}$
}

\begin{abstract}
The Papanicolaou Society of Cytopathology has developed a set of guidelines for pancreatobiliary cytology including indications for endoscopic ultrasound guided fine-needle aspiration biopsy, techniques of the endoscopic retrograde cholangiopancreatography, terminology and nomenclature of pancreatobiliary disease, ancillary testing, and postbiopsy management. All documents are based on the expertise of the authors, a review of the literature, discussions of the draft document at several national and international meetings over an 18-month period and synthesis of online comments of the draft document on the Papanicolaou Society of Cytopathology website [www.papsociety.org]. This document presents the results of these discussions regarding the use of ancillary testing in the cytological diagnosis of biliary and pancreatic lesions. This document summarizes the current state of the art for techniques in acquiring cytology specimens from the biliary tree as well as solid and cystic lesions of the pancreas. Diagn. Cytopathol. 2014;42:333-337. (c) 2014 Wiley Periodicals, Inc.
\end{abstract}

Key Words: $\quad$ pancreas; bile duct; techniques; cytology; FNA

\footnotetext{
${ }^{1}$ Division of Gastroenterology, Mass. General Hospital, 55 Fruit St. Boston, MA, USA

${ }^{2}$ Division of Gastroenterology, Indiana University

${ }^{3}$ Section of Endoscopic Oncology, Moffitt Cancer Center, Tampa, Florida

${ }^{4}$ Division of Cytopathology, Caris Life Sciences

${ }^{5}$ Division of Cytopathology, Wayne State University

${ }^{6}$ Division of Cytopathology, Yale University

${ }^{7}$ Division of Gastroenterology, University of Michigan

${ }^{8}$ Section of Cytopathology, Penn Medicine, Pennsylvania

${ }^{9}$ Division of Cytopathology, Mayo Clinic, Arizona

${ }^{10}$ Section of Cytopathology, MD Anderson Center

*Correspondence to: William Brugge, M.D., Massachusetts General Hospital, Division of Gastroenterology, Boston, MA, USA. E-mail: wbrugge@partners.org

Received 4 December 2013; Accepted 8 January 2014

DOI: $10.1002 /$ dc. 23096

Published online 19 February 2014 in Wiley Online Library (wileyonlinelibrary.com).
}

The sampling of the pancreas and biliary system for diagnostic cytology has been a major development in the diagnosis and management of patients with pancreas-biliary diseases. ${ }^{1}$ The increasing use of cytology has been made possible through the development of endoscopic techniques that provide minimally invasive tissue acquisition. Endoscopic retrograde cholangiopancreatography (ERCP) guided brush cytology of the bile duct was the initial example of providing an important diagnosis of biliary malignancy. Recently, the use of endoscopic ultrasound (EUS) guided fine-needle aspiration (FNA) has provided a supplemental technique for acquiring cytology from the pancreas and the bile duct. Through continued refinement in needles and tissue management, the diagnostic rates of FNA have improved dramatically. EUSFNA is now the procedure of choice for securing a diagnosis of a pancreas malignancy. The recent introduction of techniques for obtaining core tissue samples from the pancreas will further improve the accuracy of diagnostic cytology.

\section{Bile Ducts}

\section{Bile Duct Brushing}

Aspiration of bile duct juice during ERCP is the simplest method of obtaining a cytology specimen for the evaluation of a biliary stricture. The technique retrieves only exfoliative cells in bile and does not involve brushing. ${ }^{2}$ A simple catheter is placed into the bile duct and bile is aspirated. However, the sensitivity for this technique has been disappointing, ranging from 6 to $32 \%$ for detecting biliary malignancy. ${ }^{2,3}$ Because of the better yield of brush cytology, bile aspiration alone is rarely used to provide diagnostic cytology specimens. However, this technique can be applied to specimens collected through a chronic biliary drainage catheter. ${ }^{4}$ Cytologic analysis of 
bile duct tissue provides a more accurate diagnosis than histologic processing of tissue. ${ }^{5}$ One study suggested that the sensitivity could be improved by dilating the stricture before bile acquisition, but significant complications have been reported. ${ }^{6,7}$ Repeated brushings will improve the diagnostic yield of biliary cytology. ${ }^{8}$ Retrieved biliary stents can also be used as a source of cytologic material, but brush cytology provides a better yield of cytologic tissue. ${ }^{9}$

ERCP guided brush techniques. Biliary brush cytology is the traditional method for collecting tissue from the bile duct in the setting of a stricture. ${ }^{10}$ Standard cytology brushes are guided through a stricture, over a wire, and deployed across the stricture. The brush scrapes against the biliary mucosa and retrieves cellular material from the superficial mucosa. The brush is then retracted into a sheath and the entire device is removed from the endoscope. Cytology material is retrieved from the brush by smearing the cellular material onto a glass slide or into a fixative solution. ${ }^{11}$

Recently, a newly designed cytology brush has been introduced. The brush is $3 \mathrm{~mm}$ in diameter, $5 \mathrm{~cm}$ long, with stiffer bristles than a standard cytology brush. The bristles are oriented at 45 degrees on a $7 \mathrm{~F}$ sheath. By contrast, the standard brush is $1.5 \mathrm{~cm}$ long and has bristles oriented at 90 degrees on a $6 \mathrm{~F}$ sheath. In a study comparing each type of brush, all patients underwent sampling with each of the brushes. The cancer detection rate was not significantly different with the two brushes (27 vs. 30\%). ${ }^{12}$

Technique for tissue management. Biliary cytological material is retrieved from a brush that has been placed through a concerning stricture(s). The brush may be heavily laden with tissue, blood, and clot. Retrieval of diagnostic material from the brush should be done in the procedure room by trained endoscopy personnel.

The first step in the technique is to carefully open the brush outside of the sheath and expose the bristles and adherent tissue. Typically, the brush is placed against a glass slide and a smear of tissue is made repeatedly on several slides. After the tissue has been smeared off the brush, the brush is cut from the catheter and the brush is placed into a plastic tube containing fixative. The brush is agitated in order to dislodge additional tissue from the brush. At the completion of tissue retrieval, the brush can be removed or left within the sample tube. The slides and the sample tube are sent to cytology where the sample tube is spun to isolate the tissue for a thin prep.

Traditionally, biliary brush cytology specimens have been used solely for cytological analysis. Recently, the use of the brush cytology specimens has been expanded by using molecular markers and DNA-based testing. Although p53 and KRAS mutations are commonly seen in biliary malignancy, brush cytology specimens have not generally been used for mutation analysis. ${ }^{13}$ Biliary brush specimens for assessment of p53 mutations will require separate processing and dedicated immunostaining. ${ }^{14}$

Recently, there have been efforts to develop more objective testing of biliary cytology using image-based testing of DNA histograms for ploidy analysis. ${ }^{15}$ Digital imaging analysis and fluorescence in situ hybridization (FISH) were evaluated in a study of 233 consecutive patients undergoing ERCP for a pancreatobiliary stricture. The patients underwent standard cytology, DIA, and FISH. ${ }^{16}$ The test performance was similar across groups. Standard cytology had low sensitivity (4-20\%) but $100 \%$ specificity. In patients with negative cytology, FISH increased sensitivity while preserving specificity. The sensitivity and specificity of DIA was intermediate between routine cytology and FISH. The use of cytologic material for FISH analysis does not require alteration in specimen acquisition, except for additional material on slides.

\section{Endoscopic Forceps Biopsy of the Bile Duct}

Endoscopic forceps biopsy during ERCP is often performed in combination with brush cytology in order to improve the sensitivity of tissue sampling. ${ }^{17}$ In this technique, a small diameter forceps is placed through a widely patent ampulla and fluoroscopically directed to the area of interest in the bile duct. The biopsy specimens are processed as histologic specimens. Biopsies are often used to supplement brush cytology. One study suggested that the combination of the techniques increased the sensitivity by $\sim 15$ to $25 \%$ compared with either method alone. In a recent prospective study of 26 patients, the sensitivity, accuracy, and negative predictive values were $5.9 \%, 38.5 \%$, and $36 \%$ for standard cytology brushings, $29.4 \%, 53.8 \%$, and $42.8 \%$ for standard forceps biopsies, and $76.5 \%, 84.6 \%$, and $69.2 \%$ for miniforceps biopsies, respectively. ${ }^{18}$ When comparing the three methods of sampling, mini-forceps biopsy provided significantly better sensitivity and overall accuracy compared with standard cytology brushing and standard forceps biopsy. ${ }^{18}$ Bile duct biopsy specimens can also be smeared onto glass slides for on-site cytology analysis. ${ }^{19}$ Although malignant cytology is highly specific for a bile duct malignancy, atypia can be seen in benign inflammatory lesions. ${ }^{20}$

\section{Endoscopic Fine-Needle Aspiration of the Bile Duct}

Needle biopsy of biliary strictures and masses is performed with a biliary catheter contained as aspiration needle that be placed into the target lesion under fluoroscopic guidance. ${ }^{21}$ A combination of brush cytology and endobiliary biopsy with endoscopic FNA was more sensitive $(73-77 \%)$ than either method alone in at least three reports. ${ }^{22}$ One study suggested that combining stricture dilation, cytology, and FNA substantially improved the accuracy for diagnosis of malignant strictures caused by gallbladder or pancreatic cancer compared with cytology alone. ${ }^{23}$ 


\section{Endoscopic Cholangioscopy}

Endoscopic cholangioscopy is often performed using a dedicated, small diameter endoscope that is placed through the instrument channel for a duodenoscope. Prospective single-center case series using either endoscope-based or catheter-based systems have shown that cholangioscopic visualization with or without biopsy had a sensitivity of $89-100 \%$ and a specificity of $79-96 \%$ for detecting biliary malignancies. ${ }^{24}$ Dedicated miniforceps for cholangioscopy are very small in diameter and expensive. The small diameter forceps specimens are processed using histologic techniques.

In a recent prospective study of 26 patients who underwent sampling of a bile duct lesion using brush, standard forceps, and mini-forceps biopsy, the sensitivity, accuracy, and negative predictive values were $5.9 \%, 38.5 \%$, and $36 \%$ for standard cytology brushings, $29.4 \%, 53.8 \%$, and $42.8 \%$ for standard forceps biopsies, and $76.5 \%$, $84.6 \%$, and $69.2 \%$ for mini-forceps biopsies, respectively. ${ }^{18}$ When comparing the three methods of sampling, mini-forceps biopsy provided significantly better sensitivity and overall accuracy compared with standard cytology brushing and standard forceps biopsy. ${ }^{18}$

EUS-guided FNA of bile duct masses. Linear EUS can readily image the bile duct and associated masses from the ampulla to the birfurcation. EUS FNA of the bile duct is usually performed across the duodenum and into a focal mass arising from the bile duct. In a study of 24 consecutive patients with proximal biliary strictures (upper one-third of the bile duct) and previously nondiagnostic ERCP brush cytology, EUS visualized a mass in $23(96 \%)$ patients. $^{25}$ EUS-guided FNA demonstrated malignancy in 17 of $24(71 \%)$ of patients. The overall sensitivity, specificity, positive predictive value, negative predictive value, and accuracy of EUS-FNA were $77 \%$, $100 \%, 100 \%, 29 \%$, and $79 \%$, respectively.

Similar results were obtained in a study 81 patients with confirmed cholangiocarcinoma who underwent EUS. EUS identified the tumor in 76 patients (94\%), a rate higher than what was seen with triphasic computed tomography $(30 \%)$, or magnetic resonance imaging $(42 \%) .{ }^{26}$ EUS-FNA was performed in 74 of the patients (91\%) and diagnosed cholangiocarcinoma in 54 patients for a sensitivity of $73 \% .^{26}$ The sensitivity was higher for distal lesions than for proximal lesions ( $81 \%$ vs. $59 \%){ }^{26}$

\section{Pancreas}

\section{ERCP-Guided Brush Cytology of the Pancreatic Duct}

The yield of aspirated pancreatic juice for exfoliative cytology is very low and rarely used. ${ }^{24}$ Sampling of the main pancreatic duct can be performed with techniques similar to those used with brush cytology of the bile duct.
A wire-guided brush is used to collect cytologic material from within a strictured pancreatic duct. There are significant risks of pancreatitis with ductal brushing that can be reduced by placing a stent at the conclusion of the ERCP. ${ }^{27}$ The tissue yield of brush cytology of pancreatic duct can be improved by stricture dilation. ${ }^{28}$

\section{EUS-Guided Fine-Needle Aspiration Sampling}

Devices for EUS guided FNA. Linear endosonographic instruments are required to target lesions for FNA. ${ }^{29}$ The instrument must initially be passed through the oral pharynx, esophagus, and when necessary into the stomach and duodenum. Prior gastric surgery, such a bypass or Whipple resection, will restrict the ability of the echoendoscope to image targets adjacent to the stomach and duodenum.

The appropriate gauge EUS needle should be selected for the procedure based on the vascularity of the target lesion, the difficulty in accessing the lesion, and type of tissue needed for a diagnosis. Highly vascular lesions of the pancreas as well as uncinated lesions should be aspirated with a 25-gauge needle. The diagnosis of adenocarcinoma is best made with aspiration cytology.

Simple aspiration needles (usually 22- or 25-gauge) are used in the vast majority of targets and provide similar cytologic yield. ${ }^{30}$ Smaller gauge needles are easier to use, generally safer, and the tissue yield is higher for pancreatic adenocarcinoma. ${ }^{31}$ Because the needles pass through the mucosa of the gastrointestinal tract, there is potential for contamination with epithelial cells. A 25-gauge needle is often used for FNA of lymph nodes and vascular lesions such as suspected neuroendocrine tumors (NETs) and metastases from renal cell carcinoma. Mucinous cysts are aspirated with 22-gauge needles because of the high viscosity of the cyst fluid from IPMNs.

Core biopsy and Trucut needles (19-gauge) are used for lesions such as stromal cell tumors, NETs, tumors with suboptimal cytology yield, and for pancreatic lesions that are suspicious for autoimmune pancreatitis. Small gauge core biopsy needles have recently been made available and often used when standard aspiration techniques do not provide a diagnostic tissue. Core biopsy specimens for autoimmune pancreatitis should be processed for histology as well as for IgG4 immunostaining.

Methodology for EUS guided FNA. Under constant EUS guidance, the needle, occluded by a stylet, is placed across the gastric or duodenal wall into the target lesion. One quick thrust perpendicular to the wall of the lesion is used to enter solid lesions, rather than a slow continuous motion. Once the needle has been accurately placed into the mass lesion, the stylet is removed and suction is applied to the needle. In highly vascular lesions, minimal suction should be used. In order to maximize the yield 
from aspiration cytology, the needle is moved to and fro within the mass lesion using a fanning technique. ${ }^{32}$ The suction is then turned off, the needle removed and the specimen is placed onto the slide for processing. Additional specimens are obtained with separate passes of the needle. Cytologic interpretation of the cytology specimens on slides aids the endoscopists in obtaining specimens. The degree of vacuum suction determines the amount of aspiration tissue. Excessive suction may cause specimens to be contaminated with blood. Specimens heavily contaminated with blood may be discarded.

Smear specimens are produced on glass slides and placed in fixative, often ethanol. Cytology specimens are expressed onto slides and two smears are made. One slide is air-dried and stained with a modified Diff-Quik preparation for rapid interpretation on-site if available. The other slide is wet fixed and later stained with a modified Papanicolaou stain. Material may be obtained for cellblock preparation for later immunocytochemistry testing.

Core specimens of the pancreas are sent for histology sectioning. Large gauge needles and core biopsy needles provide a core tissue for histologic interpretation and tissue staining. Cytology specimens can be obtained from the core by rolling the cores across slides.

Whenever possible, rapid on site evaluation of cytology should be used because it reduces the frequency of falsely negative FNA, particularly in the evaluation of pancreatic masses. ${ }^{33}$ In general, sufficient needle passes will be made until diagnostic material has been secured. Without on-site cytology, approximately, 7 passes of a pancreas mass are needed to maximize the sensitivity. ${ }^{34}$ Lymph nodes can be evaluated with fewer passes, but stromal cell tumors may require $3-5$ passes. $^{34}$

False-positive and false-negative cytological diagnosis rates of pancreatic masses by EUS-FNA are low and may result from technical difficulties, sampling or interpretation errors. The false-positivity rate of EUS-FNA for a pancreatic lesion is about $2 \%$ and results from specimen contamination by an intervening mucosal malignancy or misinterpretation. ${ }^{35}$ A study of 367 patients with solid pancreatic lesions in whom EUS-FNA cytology results were interpreted as positive or suspicious for malignancy, only four cases showed chronic pancreatitis on surgical pathology. Chronic pancreatitis is also the most common benign pathology causing false-negative interpretation of a pancreatic cancer.

FNA of pancreas cystic lesions. FNA of cystic lesion involves very similar techniques as FNA of solid lesions. For suspected mucinous cysts, a 22-gauge needle is used because of the low viscosity of the fluid. Serous cystadenomas and cystic NETs should be aspirated with a 25-gauge needle in order to minimize the risk of bleeding. The cyst fluid from serous cystadenomas is thin and easily aspirated. Pseudocysts should be aspirated with a
22- or 19-gauge needle in order to evacuate the entire lesion of fluid which may become contaminated with FNA. In general, one passage of the needle should be used to evaluate a cyst and high suction will aid in the rapid emptying of the cyst. Mural nodules or adjacent masses can be aspirated separately or during the cyst fluid aspiration. At times, the nodule and mass are more apparent after evacuation of the cyst. There are reports of enhancement of the quality of cytology specimens by traversing the lining and wall of the cyst with several passes of the needle with the risk of pancreatitis and leakage. ${ }^{36}$

Aspirated cyst fluid should be carefully aliquoted for cytology, tumor markers, and DNA testing. If the primary concern is a malignancy, priority should be given to cytology. If typing of the cyst is the major clinical concern, then the fluid should sent for CEA and kRASGNAS. Cyst fluid should be centrifuged before assaying the fluid for CEA and DNA analysis.

\section{References}

1. Yoon WJ, Brugge WR. Endoscopic evaluation of bile duct strictures. Gastrointest Endosc Clin N Am 2013;23:277-293.

2. Kurzawinski TR, Deery A, Dooley JS, et al. A prospective study of biliary cytology in 100 patients with bile duct strictures. Hepatology 1993;18:1399-1403.

3. Fogel EL, Sherman S. How to improve the accuracy of diagnosis of malignant biliary strictures. Endoscopy 1999;31:758-760.

4. Hattori M, Nagino M, Ebata T, et al. Prospective study of biliary cytology in suspected perihilar cholangiocarcinoma. $\mathrm{Br} \mathrm{J}$ Surg 2011;98:704-709.

5. Ung KA, Ljung A, Wagermark $\mathrm{J}$, et al. Brush cytology is superior to biopsies obtained by a new device in bile duct strictures. Hepatogastroenterology 2007;54:664-668.

6. Mohandas KM, Swaroop VS, Gullar SU, et al. Diagnosis of malignant obstructive jaundice by bile cytology: Results improved by dilating the bile duct strictures. Gastrointest Endosc 1994;40 (Part 1):150-154.

7. Ornellas LC, Santos Gda C, Nakao FS, et al. Comparison between endoscopic brush cytology performed before and after biliary stricture dilation for cancer detection. Arq Gastroenterol 2006;43:20-23.

8. de Bellis M, Fogel EL, Sherman S, et al. Influence of stricture dilation and repeat brushing on the cancer detection rate of brush cytology in the evaluation of malignant biliary obstruction. Gastrointest Endosc 2003;58:176-182.

9. Mansfield JC, Griffin SM, Wadehra V, et al. A prospective evaluation of cytology from biliary strictures. Gut 1997;40:671-677.

10. Ferrari Junior AP, Lichtenstein DR, Slivka A, et al. Brush cytology during ERCP for the diagnosis of biliary and pancreatic malignancies. Gastrointest Endosc 1994;40 (Part 1):140-145.

11. Vadmal MS, Byrne-Semmelmeier S, Smilari TF, et al. Biliary tract brush cytology. Acta Cytol 2000;44:533-538.

12. Fogel EL, deBellis M, McHenry L, et al. Effectiveness of a new long cytology brush in the evaluation of malignant biliary obstruction: A prospective study. Gastrointest Endosc 2006;63:71-77.

13. Van Laethem JL, Bourgeois V, Parma J, et al. Relative contribution of Ki-ras gene analysis and brush cytology during ERCP for the diagnosis of biliary and pancreatic diseases. Gastrointest Endosc 1998;47:479-485.

14. Stewart CJ, Burke GM. Value of p53 immunostaining in pancreatico-biliary brush cytology specimens. Diagn Cytopathol 2000;23:308-313. 
15. Baron TH, Harewood GC, Rumalla A, et al. A prospective comparison of digital image analysis and routine cytology for the identification of malignancy in biliary tract strictures. Clin Gastroenterol Hepatol 2004;2:214-219.

16. Levy MJ, Baron TH, Clayton AC, et al. Prospective evaluation of advanced molecular markers and imaging techniques in patients with indeterminate bile duct strictures. Am J Gastroenterol 2008; 103:1263-1273.

17. Jailwala J, Fogel EL, Sherman S, et al. Triple-tissue sampling at ERCP in malignant biliary obstruction. Gastrointest Endosc 2000; 51 (Part 1):383-390.

18. Draganov PV, Chauhan S, Wagh MS, et al. Diagnostic accuracy of conventional and cholangioscopy-guided sampling of indeterminate biliary lesions at the time of ERCP: A prospective, long-term follow-up study. Gastrointest Endosc 2012;75:347-353.

19. Wright ER, Bakis G, Srinivasan R, et al. Intraprocedural tissue diagnosis during ERCP employing a new cytology preparation of forceps biopsy (Smash protocol). Am J Gastroenterol 2011;106: 294-299.

20. Glasbrenner B, Ardan M, Boeck W, et al. Prospective evaluation of brush cytology of biliary strictures during endoscopic retrograde cholangiopancreatography. Endoscopy 1999;31:712-717.

21. Howell DA, Beveridge RP, Bosco J, et al. Endoscopic needle aspiration biopsy at ERCP in the diagnosis of biliary strictures. Gastrointest Endosc 1992;38:531-535.

22. Kurzawinski T, Deery A, Davidson BR. Diagnostic value of cytology for biliary stricture. Br J Surg 1993;80:414-421.

23. Farrell RJ, Jain AK, Brandwein SL, et al. The combination of stricture dilation, endoscopic needle aspiration, and biliary brushings significantly improves diagnostic yield from malignant bile duct strictures. Gastrointest Endosc 2001;54:587-594.

24. Pugliese V, Pujic N, Saccomanno S, et al. Pancreatic intraductal sampling during ERCP in patients with chronic pancreatitis and pancreatic cancer: Cytologic studies and k-ras-2 codon 12 molecular analysis in 47 cases. Gastrointest Endosc 2001;54: 595-599.

25. DeWitt J, Misra VL, Leblanc JK, et al. EUS-guided FNA of proximal biliary strictures after negative ERCP brush cytology results. Gastrointest Endosc 2006;64:325-333.
26. Mohamadnejad M, DeWitt JM, Sherman S, et al. Role of EUS for preoperative evaluation of cholangiocarcinoma: A large singlecenter experience. Gastrointest Endosc 2011;73:71-78.

27. Vandervoort J, Soetikno RM, Montes H, et al. Accuracy and complication rate of brush cytology from bile duct versus pancreatic duct. Gastrointest Endosc 1999;49 (Part 1):322-327.

28. Yamaguchi T, Shirai Y, Nakamura N, et al. Usefulness of brush cytology combined with pancreatic juice cytology in the diagnosis of pancreatic cancer: Significance of pancreatic juice cytology after brushing. Pancreas 2012;41:1225-1229.

29. Vilmann P, Saftoiu A. Endoscopic ultrasound-guided fine needle aspiration biopsy: Equipment and technique. J Gastroenterol Hepatol 2006;21:1646-1655.

30. Lee JH, Stewart J, Ross WA, et al. Blinded prospective comparison of the performance of 22-gauge and 25-gauge needles in endoscopic ultrasound-guided fine needle aspiration of the pancreas and peri-pancreatic lesions. Dig Dis Sci 2009;54:2274-2281.

31. Madhoun MF, Wani SB, Rastogi A, et al. The diagnostic accuracy of 22-gauge and 25-gauge needles in endoscopic ultrasound-guided fine needle aspiration of solid pancreatic lesions: A meta-analysis. Endoscopy 2013;45:86-92.

32. Bang JY, Magee SH, Ramesh J, et al. Randomized trial comparing fanning with standard technique for endoscopic ultrasound-guided fine-needle aspiration of solid pancreatic mass lesions. Endoscopy 2013;45:445-450.

33. Collins BT, Murad FM, Wang JF, et al. Rapid on-site evaluation for endoscopic ultrasound-guided fine-needle biopsy of the pancreas decreases the incidence of repeat biopsy procedures. Cancer Cytopathol 2013;121:518-524.

34. LeBlanc JK, Ciaccia D, Al-Assi MT, et al. Optimal number of EUS-guided fine needle passes needed to obtain a correct diagnosis. Gastrointest Endosc 2004;59:475-481.

35. Gleeson FC, Kipp BR, Caudill JL, et al. False positive endoscopic ultrasound fine needle aspiration cytology: Incidence and risk factors. Gut 2010;59:586-593.

36. Rogart JN, Loren DE, Singu BS, et al. Cyst wall puncture and aspiration during EUS-guided fine needle aspiration may increase the diagnostic yield of mucinous cysts of the pancreas. J Clin Gastroenterol 2011;45:164-169. 